\title{
ON THE MATRIX RICCATI EQUATION ${ }^{1}$
}

\author{
J. J. LEVIN ${ }^{2}$
}

1. In this note we give several elementary results concerning the matrix Riccati equation

$$
\frac{d \Gamma}{d t}+\Gamma G_{3} \Gamma+\Gamma G_{4}-G_{1} \Gamma-G_{2}=0
$$

where $G_{1}, G_{2}, G_{3}, G_{4}$ are $n_{1} \times n_{1}, n_{1} \times n_{2}, n_{2} \times n_{1}, n_{2} \times n_{2}$ matrices respectively which are continuous on some interval $I$ (which need not be bounded or closed) and where $\Gamma$ is an $n_{1} \times n_{2}$ matrix. If $n_{1}=n_{2}=1$, then (1.1) is the classical (scalar) Riccati equation. The case $n_{1}=n_{2}$ has been considered by Radon $[6 ; 7]$, Reid $[8 ; 9]$, Sternberg $[11 ; 12]$ and Whyburn [15]. The case $n_{2}=1$ has been treated by Chiellini $[1]$ and Coles [2]. Lyaščenko $[4 ; 5]$ considers the case of arbitrary $n_{1}, n_{2}$ in connection with the problem of splitting a linear system of differential equations into two independent lower-order systems. Applications of (1.1), for $n_{1}=n_{2}$, have been made by Sternberg [13], and by Sternberg and Kaufman [14].

In $\$ 2$ an explicit formula, (2.2), for the solution of (1.1) in terms of its initial matrix and a fundamental matrix for an associated linear system of differential equations, (2.1), is given. Several theorems relating (1.1) and (2.1) are given in this section. For $n_{1}=n_{2}=1(2.2)$ is a well-known result for the scalar Riccati equation. For $n_{1}=n_{2}$ Theorem 1 is equivalent to Theorem 3.1 of [9].

In $\$ 3$ a theorem (Theorem 5) is obtained for the case $n_{1}=n_{2}=n$ which reduces when $n=1$ to the classical cross-ratio property for solutions of the scalar Riccati equation. It is different from a theorem due to Reid [9], which also reduces to the classical cross-ratio property in the scalar case, in that it involves only four solutions of the matrix Riccati equation whereas Reid's theorem involves $n^{2}+3$ solutions. The proof is, however, similar to Reid's as they both follow the classical proof, see Ince [3], and rely heavily on a theorem for linear systems due to Reid [8].

Received by the editors September 17, 1958 and, in revised form, November 21, 1958.

${ }^{1}$ The research in this document was supported jointly by the Army, Navy, and Air Force under contract with the Massachusetts Institute of Technology.

${ }^{2}$ Staff Member, Lincoln Laboratory, Massachusetts Institute of Technology. 
2. Associated with (1.1) in a basic manner is the $n_{1}+n_{2}$ dimensional linear system

$$
\frac{d}{d t} x=\left(\begin{array}{ll}
G_{1} & G_{2} \\
G_{3} & G_{4}
\end{array}\right) x
$$

where the $G_{i}$ of (2.1) are the same as in (1.1) and where $x=\operatorname{col}\left(x_{1}, \cdots, x_{n_{1}+n_{2}}\right)$. For every $s \in I$ let

$$
M(t, s)=\left(\begin{array}{ll}
M_{1}(t, s) & M_{2}(t, s) \\
M_{3}(t, s) & M_{4}(t, s)
\end{array}\right),
$$

where $M_{1}, M_{2}, M_{3}, M_{4}$ are $n_{1} \times n_{1}, n_{1} \times n_{2}, n_{2} \times n_{1}, n_{2} \times n_{2}$ matrices respectively, be the fundamental matrix for (2.1) such that $M(s, s)$ $=E_{n_{1}+n_{2}}$ (the unit $\left(n_{1}+n_{2}\right) \times\left(n_{1}+n_{2}\right)$ matrix).

THEOREM 1. Let $s \in I$ and let $\Gamma(s)$ be a given $n_{1} \times n_{2}$ matrix, then

$$
\Gamma(t)=\left[M_{1}(t, s) \Gamma(s)+M_{2}(t, s)\right]\left[M_{3}(t, s) \Gamma(s)+M_{4}(t, s)\right]^{-1}
$$

is, for $t$ sufficiently close to $s$, the solution of (1.1) having initial matrix $\Gamma(s)$ at $t=s$.

Proof. The condition $M(s, s)=E_{n_{1}+n_{2}}$ is easily seen to guarantee the local existence of the inverse matrix in (2.2). The proof is accomplished by direct substitution of (2.2) into (1.1).

Using any solution matrix of (2.1), not necessarily fundamental, a formula similar to $(2.2)$ can still be constructed. In particular, we have

THEOREM 2. Let

$$
N(t)=\left(\begin{array}{ll}
N_{1}(t) & N_{2}(t) \\
N_{3}(t) & N_{4}(t)
\end{array}\right)
$$

where $N_{1}, N_{2}, N_{3}, N_{4}$ are $n_{1} \times n_{1}, n_{1} \times n_{2}, n_{2} \times n_{1}, n_{2} \times n_{2}$ matrices respectively, be a solution matrix for (2.1). If $C$ is a constant $n_{1} \times n_{2}$ matrix, then

$$
\Gamma(t)=\left[N_{1}(t) C+N_{2}(t)\right]\left[N_{3}(t) C+N_{4}(t)\right]^{-1}
$$

is a solution of (1.1) whenever it exists.

Proof. Substitute (2.3) into (1.1).

In the scalar case it is well known that, given any four continuously differentiable functions $N_{i}(t)(i=1,2,3,4)$ such that $N_{1} N_{4}-$ $N_{2} N_{3} \neq 0,(2.3)$ defines a Riccati equation and is a solution of it for any constant $C$. The following theorem is a generalization of this result. 
Theorem 3. Let $N_{1}(t), N_{2}(t), N_{3}(t), N_{4}(t)$ be $n_{1} \times n_{1}, n_{1} \times n_{2}, n_{2} \times n_{1}$, $n_{2} \times n_{2}$ continuously differentiable matrices on $I$ and let

$$
N(t)=\left(\begin{array}{ll}
N_{1}(t) & N_{2}(t) \\
N_{3}(t) & N_{4}(t)
\end{array}\right)
$$

be nonsingular on $I$. Then

$$
\Gamma(t)=\left[N_{1}(t) C+N_{2}(t)\right]\left[N_{3}(t) C+N_{4}(t)\right]^{-1}
$$

where $C$ is a constant $n_{1} \times n_{2}$ matrix, defines a matrix Riccati equation and is a solution of it whenever (2.4) exists.

Proof. Define $G(t)=d N(t) / d t N^{-1}(t)$. Then $N(t)$ is a fundamental matrix for $d x / d t=G(t) x$. Partition $G(t)$ so that

$$
G(t)=\left(\begin{array}{ll}
G_{1}(t) & G_{2}(t) \\
G_{3}(t) & G_{4}(t)
\end{array}\right)
$$

where the $G_{i}$ of (2.5) are of the same size as the $G_{i}$ of (1.1). Defining a matrix Riccati equation of the form (1.1) with the $G_{i}$ of (2.5) and applying the preceding theorem completes the proof.

One of the applications of the matrix Riccati equation concerns the local behavior of solutions of nonlinear systems of differential equations. In this connection (2.1) would arise as the first variational equation of a nonlinear system of differential equations with respect to some base solution. Formula (2.2) then plays the following role.

THEOREM 4. Let $s \in I$ and let $\Gamma(s)$ be a given $n_{1} \times n_{2}$ matrix. If

$$
\operatorname{col}\left(x_{1}(t), \cdots, x_{n_{1}+n_{2}}(t)\right)
$$

is any solution of (2.1) such that

$$
\operatorname{col}\left(x_{1}(s), \cdots, x_{n_{1}}(s)\right)=\Gamma(s) \operatorname{col}\left(x_{n_{1}+1}(s), \cdots, x_{n_{1}+n_{2}}(s)\right),
$$

then

$$
\operatorname{col}\left(x_{1}(t), \cdots, x_{n_{1}}(t)\right)=\Gamma(t) \operatorname{col}\left(x_{n_{1}+1}(t), \cdots, x_{n_{1}+n_{2}}(t)\right)
$$

for $t$ sufficiently close to $s$, where $\Gamma(t)$ is given by (2.2).

Proof. It is easily seen that

$$
\begin{aligned}
& \operatorname{col}\left(x_{1}(t), \cdots, x_{n_{1}}(t)\right) \\
& \quad=\left[M_{1}(t, s) \Gamma(s)+M_{2}(t, s)\right] \operatorname{col}\left(x_{n_{1}+1}(s), \cdots, x_{n_{1}+n_{2}}(s)\right), \\
& \begin{aligned}
\operatorname{col}\left(x_{n_{1}+1}(t), \cdots,\right. & \left.x_{n_{1}+n_{2}}(t)\right) \\
& =\left[M_{3}(t, s) \Gamma(s)+M_{4}(t, s)\right] \operatorname{col}\left(x_{n_{1}+1}(s), \cdots, x_{n_{1}+n_{2}}(s)\right)
\end{aligned}
\end{aligned}
$$


if $\operatorname{col}\left(x_{1}(t), \cdots, x_{n_{1}+n_{2}}(t)\right)$ is any solution of (2.1) that satisfies the hypothesis. Eliminating $\operatorname{col}\left(x_{n_{1}+1}(s), \cdots, x_{n_{1}+n_{2}}(s)\right)$ from these equations in the obvious manner completes the proof.

3. In this section $n_{1}=n_{2}=n$. The following result on linear systems, due to Reid [8], is used in the proof of the next theorem.

Lemma. Let $A(t)$ and $B(t)$ be $n \times n$ continuous matrices for $t \in I$. Let $\Lambda(t)$ and $\Omega(t)$ be $n \times n$ nonsingular matrices that satisfy

$$
\frac{d \Lambda}{d t}=A \Lambda, \quad \frac{d \Omega}{d t}=\Omega B \quad(t \in I) .
$$

Then $P(t)$ satisfies

$$
\frac{d P}{d t}=A P+P B
$$

if and only if there exists a constant matrix $C$ such that

$$
P(t)=\Lambda(t) C \Omega(t)
$$

Theorem 5. If $\Gamma_{1}, \Gamma_{2}, \Gamma_{3}, \Gamma_{4}$ are solutions of (1.1) that are continuous on some common interval $I^{*} \subset I$, and if there exists a $t_{0} \in I^{*}$ such that $\Gamma_{i}\left(t_{0}\right)-\Gamma_{j}\left(t_{0}\right)$ is nonsingular for $i \neq j$, then

(3.1) $\left(\Gamma_{3}-\Gamma_{1}\right)^{-1}\left(\Gamma_{3}-\Gamma_{2}\right)\left(\Gamma_{4}-\Gamma_{2}\right)^{-1}\left(\Gamma_{4}-\Gamma_{1}\right)=\Lambda K \Lambda^{-1} \quad\left(t \in I^{*}\right)$ where $\Lambda=\Lambda(t)$ and $K$ (a constant matrix) are nonsingular matrices.

Proof. Define

$$
U_{i}=\Gamma_{i+1}-\Gamma_{1} \quad(i=1,2,3) .
$$

Substitution shows that the $U_{i}$ are solutions of

$$
\frac{d U}{d t}+U G_{3} U+\left(\Gamma_{1} G_{3}-G_{1}\right) U+U\left(G_{3} \Gamma_{1}+G_{4}\right)=0 .
$$

Hence, the $U_{i}$ satisfy $d U / d t=A U+U B$, where $A=-U G_{3}-\Gamma_{1} G_{3}+G_{1}$, $B=-G_{3} \Gamma_{1}-G_{4}$. Therefore, as $U_{i}\left(t_{0}\right)$ is nonsingular by hypothesis, it follows from the preceding lemma that $U_{i}(t)$ is nonsingular for $t \in I^{*}$. A similar calculation shows that $U_{i}(t)-U_{j}(t)(i \neq j)$ is nonsingular for $t \in I^{*}$. Define

$$
V_{i}=U_{i}^{-1} \quad(i=1,2,3) .
$$

Substitution shows that the $V_{i}$ are solutions of

$$
d V / d t=\left(G_{3} \Gamma_{1}+G_{4}\right) V+V\left(\Gamma_{1} G_{3}-G_{1}\right)+G_{3} .
$$


Define

$$
W_{i}=V_{i+1}-V_{1} \quad(i=1,2) .
$$

Then the $W_{i}$ are solutions of

$$
d W / d t=\left(G_{3} \Gamma_{1}+G_{4}\right) W+W\left(\Gamma_{1} G_{3}-G_{1}\right) .
$$

As

$$
W_{i}=V_{i+1}-V_{1}=U_{i+1}^{-1}-U_{1}^{-1}=U_{i+1}^{-1}\left(U_{1}-U_{i+1}\right) U_{1}^{-1} \quad(i=1,2)
$$

we see that the $W_{i}$ are nonsingular for $t \in I^{*}$. Hence, by the preceding lemma, there exist nonsingular constant matrices $C_{1}$ and $C_{2}$ such that

$$
W_{i}=\Lambda C_{i} \Omega \quad\left(t \in I^{*}\right),(i=1,2)
$$

where $\Lambda$ and $\Omega$ are nonsingular matrices that satisfy

$$
\frac{d \Lambda}{d t}=\left(G_{3} \Gamma_{1}+G_{4}\right) \Lambda, \quad \frac{d \Omega}{d t}=\Omega\left(\Gamma_{1} G_{3}-G_{1}\right) .
$$

From (3.2), (3.3), (3.4), and (3.5) one has

$$
\left(\Gamma_{i+2}-\Gamma_{1}\right)^{-1}-\left(\Gamma_{2}-\Gamma_{1}\right)^{-1}=\Lambda C_{i} \Omega \quad(i=1,2) .
$$

Multiplying (3.6) by $\Gamma_{2}-\Gamma_{1}$ on the right and by $\Gamma_{i+2}-\Gamma_{1}$ on the left yields

$$
\Gamma_{2}-\Gamma_{i+2}=\left(\Gamma_{i+2}-\Gamma_{1}\right) \Lambda C_{i} \Omega\left(\Gamma_{2}-\Gamma_{1}\right) \quad(i=1,2) .
$$

Hence, as $\Gamma_{2}-\Gamma_{3}$ and $\Gamma_{2}-\Gamma_{4}$ are nonsingular,

$$
\begin{aligned}
\left(\Gamma_{2}-\Gamma_{3}\right)^{-1}\left(\Gamma_{3}-\Gamma_{1}\right) \Lambda C_{1} \Omega\left(\Gamma_{2}\right. & \left.-\Gamma_{1}\right) \\
& =\left(\Gamma_{2}-\Gamma_{4}\right)^{-1}\left(\Gamma_{4}-\Gamma_{1}\right) \Lambda C_{2} \Omega\left(\Gamma_{2}-\Gamma_{1}\right)
\end{aligned}
$$

from which (3.1), with $K=C_{1} C_{2}^{-1}$, follows easily.

\section{BIBLIOGRAPHY}

1. A. Chiellini, Sui sistemi di Riccati, Rend. Sem. Sci. Univ. Cagliari vol. 18 (1948) pp. 44-58. 338.

2. W. J. Coles, Linear and Riccati systems, Duke Math. J. vol. 22 (1955) pp. 333-

3. E. L. Ince, Ordinary differential equations, London, 1927.

4. N. Ya. Lyaščnko, On a separation theorem for a system of linear differential equations, Dokl. Akad. Nauk SSSR (N.S.) vol. 97 (1954) pp. 965-967.

5. - On a theorem of complete separation of a linear homogeneous system of ordinary differential equations and some properties of the separation matrix, Ukrain. Mat. Ž. vol. 7 (1955) pp. 403-418.

6. J. Radon, Über die Oszillationstheoreme der konjugierten Punkte beim Probleme von Lagrange, Bayer. Akad. Wiss. Math.-Nat. Kl. Abh. (1927) pp. 243-257. 
7. —_ Zum Problem von Lagrange, 4 Vorträge, Gehalten in Mathematischen Seminar der Hamburgischen Universität, July 7-24, 1928, Abhandlungen, Hamburg, vol. 6, pp. 273-299.

8. W. T. Reid, Some remarks on linear differential systems, Bull. Amer. Math. Soc. vol. 45 (1939) pp. 414-419.

9. - - A matrix differential equation of Riccati type, Amer. J. Math. vol. 68 (1946) pp. 237-246.

10. - - Addendum to a matrix differential equation of Riccati type, Amer. J. Math. vol. 70 (1948) p. 460.

11. R. L. Sternberg, Variational methods and nonoscillation theorems for systems of differential equations, Duke Math. J. vol. 19 (1952) pp. 311-322.

12. - A theorem on Hermitian solutions for related matrix differential and integral equations, Portugal. Math. vol. 12 (1953) pp. 135-139.

13. - Applications of the theory of differential equations to vibrating beams, Portugal. Math. vol. 13 (1954) pp. 111-120.

14. R. L. Sternberg and H. Kaufman, Applications of the theory of systems of differential equations to multiple non-uniform transmission lines, J. Math. Phys. (1953) pp. 244-252.

15. W. M. Whyburn, Matrix differential equations, Amer. J. Math. vol. 56 (1934) pp. 587-592.

Massachusetts Institute of Technology 